\title{
Assessment of Material Dislocation Damage by Nonlinear Ultrasound
}

\author{
Zaifu Zhan1, Shen Wang ${ }^{1, a}$, Songling Huang ${ }^{1}$, Yang Zheng ${ }^{2}$, Fuping Wang ${ }^{1}$ and Wei Zhao ${ }^{1}$ \\ ${ }^{1}$ State Key Laboratory of Power System, Department of Electrical Engineering, Tsinghua University, 100084 Beijing, China \\ ${ }^{2}$ Inspection and Research Institute, China Special Equipment, 100084 Beijing, China
}

\begin{abstract}
Under harsh environment or during service, the mechanical properties of materials or structure will deteriorate. Most of the simulations exhibit the phenomenon of nonlinearity by introducing the actual small defects, without considering dislocation. In this manuscript, subroutines are written to change the mechanical constitutive behaviour of materials. When the mechanical constitutive behaviour of the material is not linear any more, it is found that the propagation of ultrasonic wave in the material will show more obvious nonlinear phenomenon. Furthermore, the nonlinear detection coefficient is used to characterize the increase of harmonic components. This work provides a new idea for nonlinear ultrasonic testing.
\end{abstract}

\section{Introduction}

Since structure under periodic load or in harsh environment, no matter what materials, will undergo material properties degradation. Continuous degradation leads to fail of structures, sometimes with serious consequences. The evaluation of structural changes of materials and constructions and monitoring their ultimate strength and endurance in operation is required for many industrial structural integrity problems. Especially the evaluation of accumulated damage or degradation in material properties at the early stage of fracture is important in refinery plants, nuclear power plants, or aircraft parts ${ }^{[1-2]}$.

The early degradation of the mechanical properties takes up most of the life of the structures. Especially, the research shows that the degradation process includes the accumulation of the dislocation inside the materials, formation of the persistent slip band and finally the appearance of the cracks. This process accounts for $80 \% \sim 90 \%{ }^{[3-4]}$ of the life of the whole structure when the metal material structure is bearing periodic load. Therefore, testing of the early properties of materials is particularly important.

The most powerful nondestructive way of evaluating material degradation is the ultrasonic method since the characteristics of ultrasonic wave propagation are directly related to the properties of the material. Traditional ultrasonic nondestructive evaluation (NDE) is based on linear theory and normally relies on measuring some particular parameter (sound velocity, attenuation, transmission and reflection coefficients) of the propagating signal to determine the elastic properties of a material or to detect defects ${ }^{[5]}$. The presence of defects changes the phase and/or amplitude of the output signal,

\footnotetext{
${ }^{\mathrm{a}}$ Corresponding author: wangshen@mail.tsinghua.edu.cn
}

but the frequency of the input and output signals is the same.

However, the conventional ultrasonic technique is sensitive to gross defects or opens cracks, where there is an effective barrier to transmission, whereas it is less sensitive to evenly distributed microcracks or degradation $^{[6]}$. An alternative technique to overcome this limitation is nonlinear ultrasonics ${ }^{[7-12]}$. The principal difference between linear and non-linear ultrasound NDE is that in the latter the existence and characteristics of defects are often related to an acoustic signal whose frequency differs from that of the input signal. This is related to the radiation and propagation of finite amplitude (especially high power) ultrasound and its interaction with discontinuities, such as cracks, interfaces and voids. Since material failure or degradation is usually preceded by some kind of nonlinear mechanical behaviour before significant plastic deformation or material damage occurs, considerable attention has recently been focused on the application of nonlinear ultrasonics ${ }^{[13]}$.

When the material is undamaged, it obeys the linear constitutive relation. The wave propagation in the material is linear. But when the material is damaged, the mechanical constitutive behaviours is no longer linear any more. The constitutive relation of damaged materials is extremely complex ${ }^{[12-15]}$. This led to distortion of wave form and appearance of harmonics in the process of wave propagation.

Many researchers have done the research in nonlinearities of the structure response after ultrasonic excitation. Buck ${ }^{[16]}$ studied this theoretically and experimentally, and developed a pinned dislocation monopoles model of ultrasonic and dislocation effects. He pointed out that the generation of ultrasonic second harmonic depends on the displacement movement of a single dislocation between the pinning points which are 
under stress. The theoretical formulas among the amplitude of the second harmonic, the stress and the length of the dislocation line are derived. Cantrell[17] et al proposed a new model of ultrasonic to interact with dislocation dipoles and dipole arrays for exciting second harmonics. They believed that when the dislocation is dense, the amplitude of the second harmonic of the ultrasound is not only related to the length and stress of the dislocation lines, but also largely influenced by the arrangement of dislocations in the structure.

However, few researchers simulate the interact between microscopic dislocations and ultrasound. Most scholars ignore this earlier dislocation stage and study crack directly, like Ref $[2,3,6,8,18,19,21]$. This manuscript simulates the damage before cracks appear and conclude that ultrasound can already reflect early dislocations of material damage, which will be an important guarantee for NDE in military and aerospace.

The remainder of this manuscript is organized as follows: Section 2 introduces not only the basic theoretical background of nonlinear ultrasound and higher harmonics generation in theory but also constitutive behaviour which are written for simulations. In Section 3, a finite element model(FEM) of the propagation of nonlinear longitudinal waves is described in detail. FEM simulation results are presented and discussed in Section 4, and conclusions are drawn in Section 5.

\section{Basic Theoretical Background}

In this section, the nonlinear wave equation is derived from two methods. It can be seen from the derivation that if the material no longer satisfies the linear Hooke's law, the wave equation is also nonlinear, which leads to the generation of nonlinear phenomena. Therefore, the constitutive relationship is changed in simulation for damaged materials. The changed constitutive relation also will be given.

\subsection{Nonlinear wave equation}

This is the general expression for the free energy of a deformed isotropic body [14]:

$$
F=F_{0}+\frac{1}{2} \lambda \varepsilon_{\mathrm{ii}}^{2}+\mu \varepsilon_{\mathrm{ik}}^{2}
$$

where, the coefficients $\lambda$ and $\mu$ are called Lamé coefficients, $F_{0}$ is the initial free energy value, $\varepsilon_{i k}$ is the strain tensor whose linear expression is ${ }^{[14]}$ :

$$
\varepsilon_{\mathrm{ik}}=\frac{1}{2}\left(\frac{\partial u_{\mathrm{i}}}{\partial x_{\mathrm{k}}}+\frac{\partial u_{\mathrm{k}}}{\partial x_{\mathrm{i}}}\right)
$$

where, $u_{\mathrm{i}}$ is particle displacement in $i$ direction and $x_{1}, x_{2}$ and $x_{3}$ are $x, y, z$ directions respectively in Cartesian coordinate system.

When we consider nonlinearity, the strain tensor should be expanded ${ }^{[14]}$ :

$$
\varepsilon_{\mathrm{ik}}=\frac{1}{2}\left(\frac{\partial u_{\mathrm{i}}}{\partial x_{\mathrm{k}}}+\frac{\partial u_{\mathrm{k}}}{\partial x_{\mathrm{i}}}+\frac{\partial u_{1}}{\partial x_{\mathrm{k}}} \frac{\partial u_{1}}{\partial x_{\mathrm{i}}}\right)
$$

If only one-dimensional situation is considered, the linear wave equation:

$$
\frac{\partial^{2} u}{\partial t^{2}}-c \frac{\partial^{2} u}{\partial x^{2}}=0
$$

should be amended to:

$$
\frac{\partial^{2} u}{\partial t^{2}}-c\left(1-\beta \frac{\partial u}{\partial x}+\epsilon\left(\frac{\partial u}{\partial x}\right)^{2}\right) \frac{\partial^{2} u}{\partial x^{2}}=0
$$

where, $c$ is wave celocity, $\beta$ and $\epsilon$ are the quadratic and cubic nonlinear coefficients, respectively.

There is another simpler theoretical explanation that when the material is undamaged, the relationship between stress and strain is linear:

$$
\sigma=E \varepsilon
$$

However, when the material is damaged, the relationship between stress and strain is no longer linear:

$$
\sigma=E \varepsilon(1+\beta \varepsilon+\ldots)
$$

where, $\sigma$ is stress. Substituting this relation into onedirectional wave equation:

$$
\rho \frac{\partial^{2} u}{\partial t^{2}}=\frac{\partial \sigma}{\partial x}
$$

Then we can obtain nonlinear wave equation:

$$
\rho \frac{\partial^{2} u}{\partial t^{2}}=A \frac{\partial u}{\partial x}+B \frac{\partial u}{\partial x} \frac{\partial^{2} u}{\partial x^{2}}
$$

Cantrell $[4,20]$ et al. also established similar nonlinear ultrasonic wave equation:

$$
\rho \frac{\partial^{2} u}{\partial t^{2}}=k_{2} \frac{\partial u}{\partial x}+\left(3 k_{2}+k_{3}\right) \frac{\partial u}{\partial x} \frac{\partial^{2} u}{\partial x^{2}}
$$

\subsection{Mechanical constitutive behaviour}

If we only consider the $x$ direction, the stress-strain relationship obeys eq. (6). Its components can be expressed in the form of:

$$
\sigma_{\mathrm{ij}}=\lambda \delta_{\mathrm{ij}} \varepsilon_{\mathrm{kk}}+2 \mu \varepsilon_{\mathrm{ij}}
$$

where $\delta_{\mathrm{ij}}$ is the Kronecker Symbol, $\delta_{\mathrm{ij}}=1$ if $\mathrm{i}=\mathrm{j}$ and $\delta_{\mathrm{ij}}=0$ if $\mathrm{i} \neq \mathrm{j}$.

For simplicity, we only consider the quadratic constitutive relation:

$$
\sigma=E \varepsilon(1+\beta \varepsilon)
$$

The code of subroutine VUMAT needs the form of component, which is

$$
\sigma_{\mathrm{ij}}=\left(\lambda \delta_{\mathrm{ij}} \varepsilon_{\mathrm{kk}}+2 \mu \varepsilon_{\mathrm{ij}}\right)\left(1+\beta \lambda \delta_{\mathrm{ij}} \varepsilon_{\mathrm{kk}}+2 \beta \mu \varepsilon_{\mathrm{ij}}\right)
$$

Because the Explicit solver of Abaqus software will be used in the following simulation, recursive eq. (14) is required. Other details are described in the next section.

$$
\Delta \sigma_{\mathrm{ij}}=\left(\lambda \delta_{\mathrm{ij}} \Delta \varepsilon_{\mathrm{kk}}+2 \mu \Delta \varepsilon_{\mathrm{ij}}\right)\left(1+\beta \lambda \delta_{\mathrm{ij}} \Delta \varepsilon_{\mathrm{kk}}+2 \beta \mu \Delta \varepsilon_{\mathrm{ij}}\right)
$$


In this manuscript, the following work will begin with the linear stress-strain relation of materials and change the stress-strain relation in materials by writing subroutines, so as to simulate the undamaged and damaged materials.

\section{Numerical Model}

Two-dimensional FE models were built with Abaqus and dynamic simulations were conducted with Explicit solver.

The Explicit solver is specialized in solving complex problems such as Explicit dynamic analysis within short time. It does not solve the coupled equation of system or generate the total stiffness matrix in each increment step, but recurs results of the previous step in a small increment step in time domain. It only calculates the total energy in the simulation to determine whether there is divergence in each increment step. So we need the recurrence eq. (14) of constitutive relation in the second section.

Abaqus software provides a user Subroutine interfaceVUMAT for User to define the mechanical constitutive behaviour of a material. Therefore, the constitutive relationship of materials is changed by writing user subroutine VUMAT, in which $\beta$ is set to $0,0.0001,0.0002$, $0.0003,0.0004$ and 0.0005 respectively for simplicity. When $\beta$ equals 0 , it means the material is undamaged.

The $2 \mathrm{D}$ model is $40 \mathrm{~mm}$ long and $20 \mathrm{~mm}$ thickness. The excitation is applied in the left edge of model within the width of $6 \mathrm{~mm}$, and the excitation amplitude is $6 \mu \mathrm{m}$, as shown in Fig. 1. The material properties are in table 1.

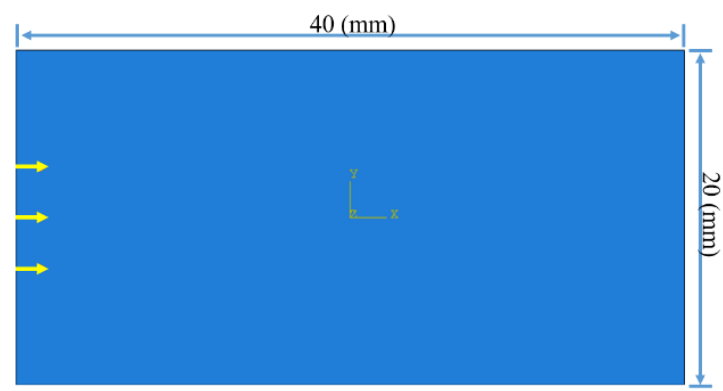

Figure 1. 2D model and excitation.

Table 1. Material properties.

\begin{tabular}{|c|c|c|c|}
\hline $\begin{array}{c}\text { Material } \\
\text { type }\end{array}$ & Density & $\begin{array}{c}\text { Young's } \\
\text { modulus }\end{array}$ & $\begin{array}{c}\text { Poisson's } \\
\text { ratio }\end{array}$ \\
\hline Steel & $7800 \mathrm{~kg} / \mathrm{m}^{3}$ & $2 \times 10^{11} \mathrm{~Pa}$ & 0.3 \\
\hline
\end{tabular}

The excitation signal shown in Fig. 2 is a five cycle tone-burst. Window function is used to prevent spectrum leakage. The excitation signal central frequency is $3 \times 10^{6}$ $\mathrm{Hz}$, and its expression is as follows:

$$
y=\sin (2 \pi f t)(0.08+0.46(1-\cos (2 \pi f t / 5)))
$$

The mesh size must be able to represent the physical characteristics of longitudinal wave propagation. So the smallest wavelength $\lambda_{\min }$ must be correctly sampled to
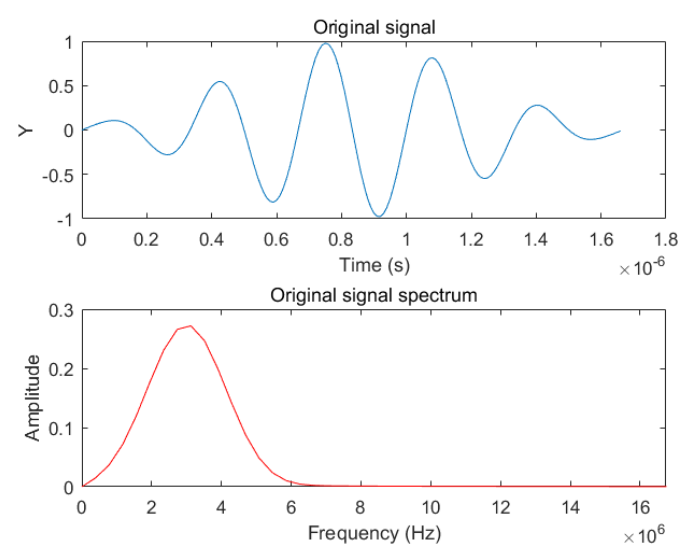

Figure 2. Excitation signal and spectrum.

describe the propagating mode in the frequency range for analysis. The spatial discretization of $\delta x_{1}$ and $\delta x_{2}$ of each element of the mesh satisfies the condition ${ }^{[21]}$.

$$
\frac{\lambda_{\min }}{\max \left(\delta x_{1}, \delta x_{2}\right)}>10
$$

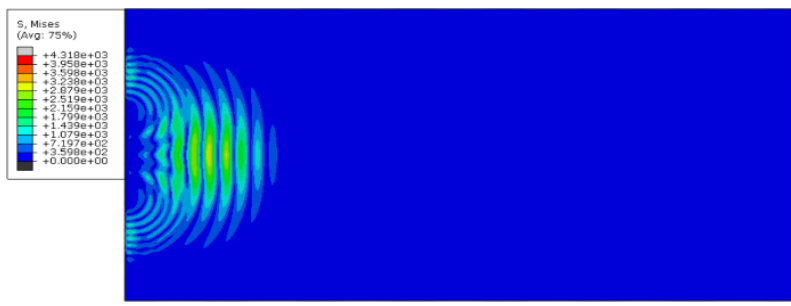

(a) $\mathrm{t}=1.697 \times 10^{-6} \mathrm{~s}$.

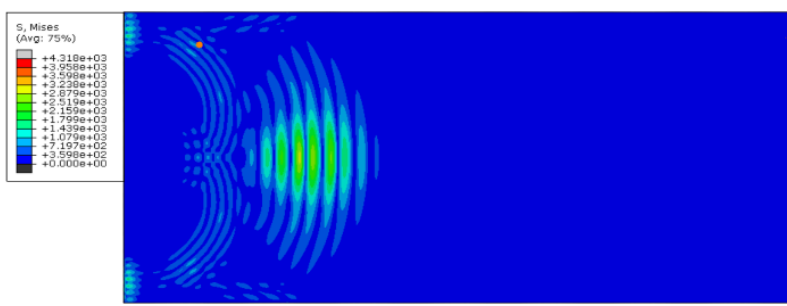

(b) $\mathrm{t}=2.769 \times 10^{-6} \mathrm{~s}$.

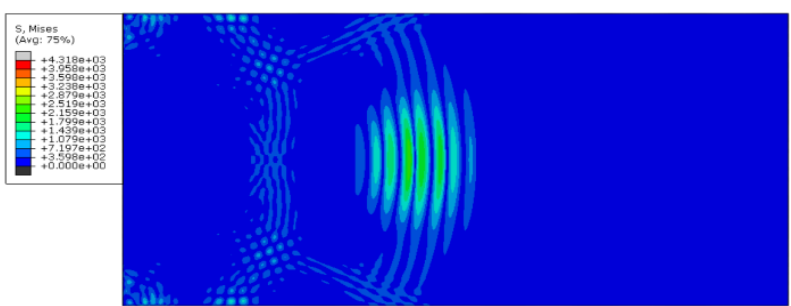

(c) $\mathrm{t}=3.889 \times 10^{-6} \mathrm{~s}$.

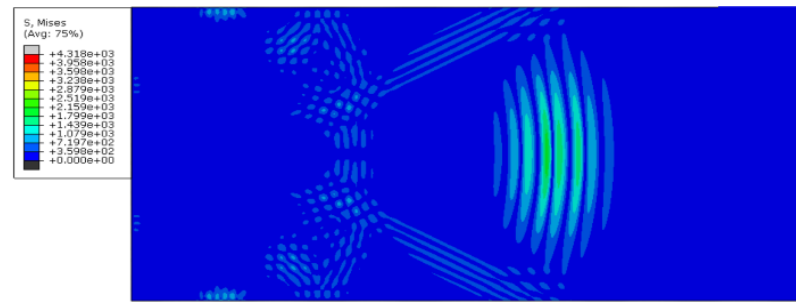

(d) $\mathrm{t}=5.2 \times 10^{-6} \mathrm{~s}$

Figure 3. Stress nephogram at different times during ultrasonic propagation. 
Due to the need to measure harmonics, the size of mesh elements is set to $5 \times 10^{-5} \mathrm{~m}$.

Abaqus software contains a wealth of mesh elements libraries. We choose CPE4R (i.e. linear reduction integral plane strain type) as the element type. It should be noted that the linear reduced integral refers to the linear interpolation used by the mesh elements in the calculation, which has no relations with the nonlinear source mentioned in this manuscript, but only for saving the calculation resources and meeting the algorithm of Abaqus/Explicit solver.

Another stability condition is that the time step must be chosen so that no wave can propagate across one mesh spacing in less than one-time step. Typically, the time discretization must satisfy the condition ${ }^{[21]}$ :

$$
\Delta t \leq 0.7 \frac{\min \left(\delta x_{1}, \delta x_{2}\right)}{c_{1}}
$$

where $c_{1}$ is the velocity of longitudinal wave. Therefore, in order to be precise, the step time is set as $1 \mathrm{~ns}$, and the total simulation time is $8 \mu \mathrm{s}$.

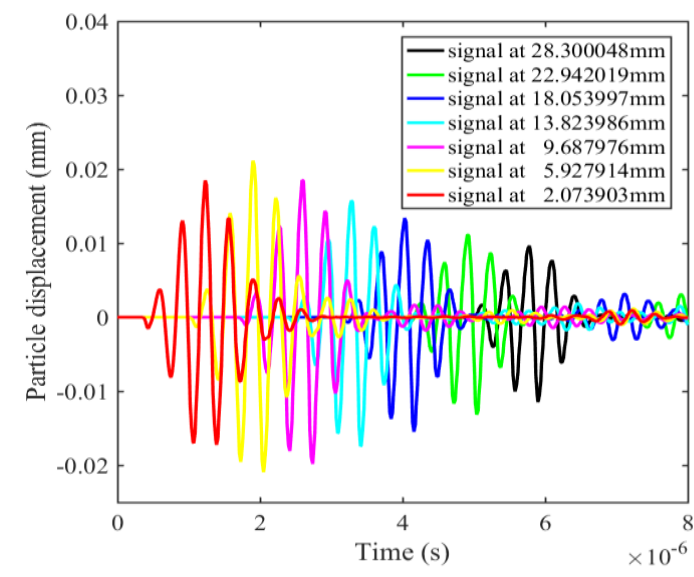

Figure 4. Waveform at different position in undamaged material model.

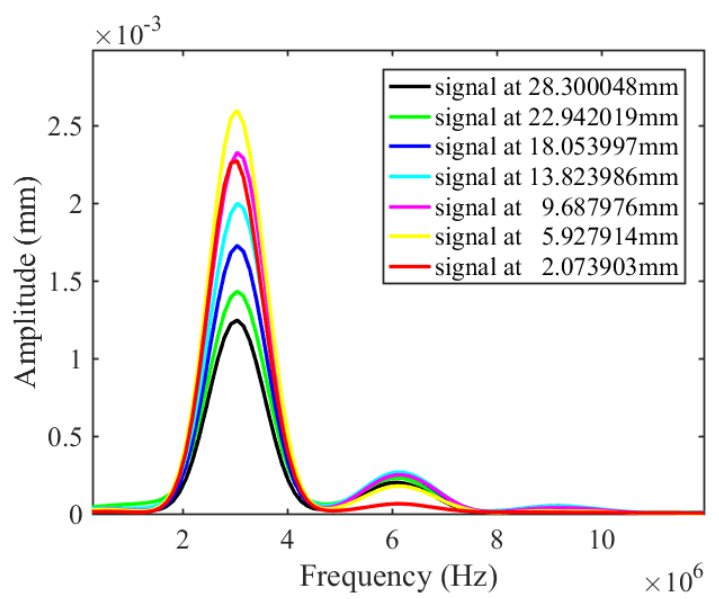

Figure 5. Spectrum of each curve in Fig. 4.

\section{Simulation Result and Discussion}

The wave propagation process is shown in Fig. 3 with stress nephogram for more intuitive. In the process of ultrasonic propagation, the displacement boundary condition excites not only the longitudinal wave but also the transverse wave. The propagation speed of transverse wave is obviously lower than longitudinal wave.

When the material is not damaged in simulations(i.e. $\beta$ is set to zero or simulate without VUMAT), the constitutive relation in the material is linear, such as eq. (6) or eq. (11). Select several points on the propagation path of the material, and take the curve of the strain component $\varepsilon_{11}$ (because the wave is a longitudinal wave) as shown in Fig. 4. In Fig. 5, spectrum analysis on each curve(signals at different distance) were performed for obtaining the fundamental, second and third harmonic components, as shown in the table 2. Combined with the stress nephogram in Fig. 3, we can clearly see that, in the process of wave propagation, the width of the wave is widened and the beam spreading phenomenon appears. Therefore, with the increase of the propagation distance, the energy is dispersed, and the particle displacement of the measurement point decreases with wave propagation.

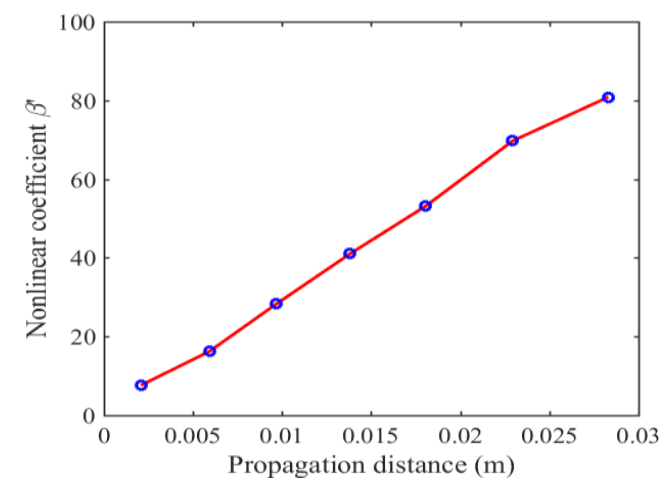

Figure 6. Variation of nonlinear detection coefficient $\beta^{\prime}$ with propagation distance in undamaged material.

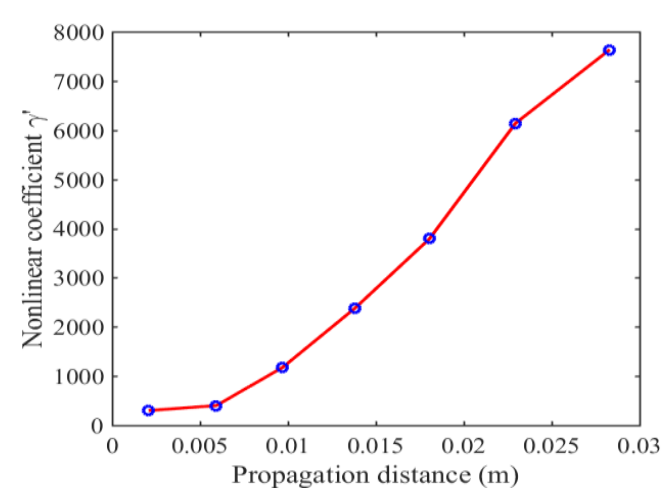

Figure 7. Variation of nonlinear detection coefficient $\gamma^{\prime}$ with propagation distance in undamaged material. 
Table 2. Harmonic components at different positions.

\begin{tabular}{|c|c|c|c|c|c|c|c|}
\hline Propagation distance $(\mathrm{mm})$ & 2.073903 & 5.927914 & 9.687976 & 13.823986 & 18.053997 & 22.942019 & 28.300048 \\
\hline Baseband amplitude $(\mathrm{mm})$ & 0.003605 & 0.004121 & 0.003691 & 0.003171 & 0.00274 & 0.002272 & 0.001977 \\
\hline Second harmonic amplitude $(\mathrm{mm})$ & 0.000099 & 0.000276 & 0.000386 & 0.000413 & 0.000399 & 0.00036 & 0.000316 \\
\hline Third harmonic amplitude $(\mathrm{mm})$ & 0.000014 & 0.000028 & 0.000059 & 0.000076 & 0.000078 & 0.000072 & 0.000059 \\
\hline
\end{tabular}

In Fig. 4, we can find that there is small vibration after five periods of longitudinal wave propagation. The main reason is the extra transverse wave effect. Although there are additional shear waves, the shear wave frequency is the same as the original excitation frequency, so it will not affect the frequency analysis.

However, due to the phenomenon of beam spreading in the process of propagation, we cannot directly compare the harmonic magnitude. We need to further analyse by introducing $\beta^{\prime}$ and $\gamma^{\prime}$ of nonlinear ultrasonic detection coefficient, the expression is as follows ${ }^{[18]}$ :

$$
\begin{gathered}
\beta^{\prime}=\frac{A_{2}}{A_{1}^{2}} \\
\gamma^{\prime}=\frac{A_{3}}{A_{1}^{3}}
\end{gathered}
$$

where, $A_{\mathrm{i}}$ is the amplitude of the $\mathrm{i}^{\text {th }}$ harmonic.

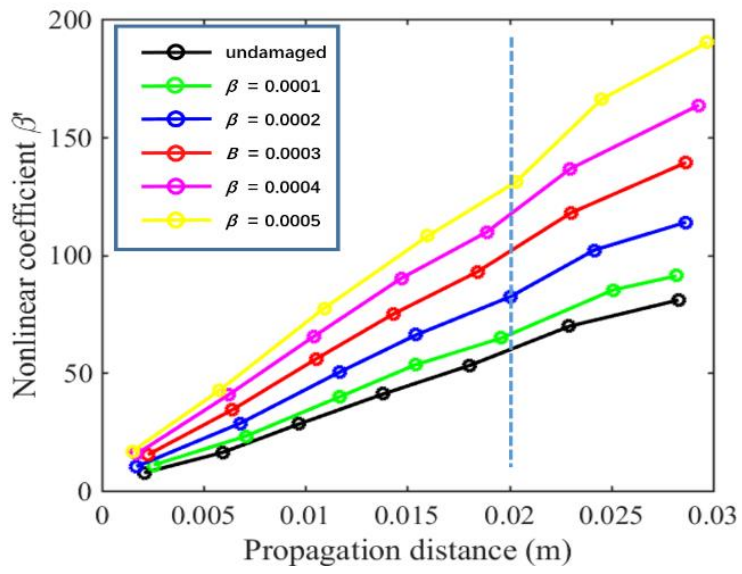

Figure 8. Variation of nonlinear detection coefficient $\beta^{\prime}$ with propagation distance in different damaged degree models.

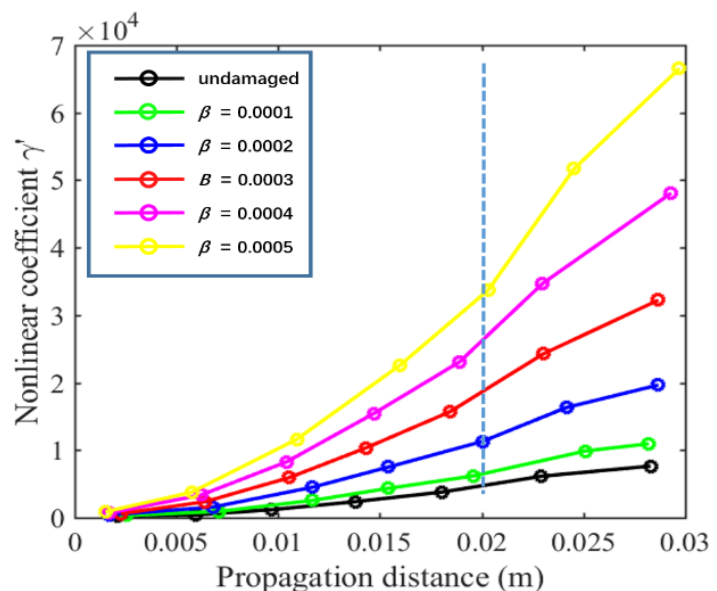

Figure 9. Variation of nonlinear detection coefficient $\gamma^{\prime}$ with propagation distance in different damaged degree models.
We analyze the spectrum of each curve data from table 2 and get the result as shown in Fig. 6 and Fig. 7. We can see that the nonlinear detection coefficient $\beta^{\prime}$ and $\gamma^{\prime}$ increases with the increase of propagation distance. This reflects the accumulation of harmonics in the process of propagation.

When we change the constitutive relation in materials, such as eq. (12). We have done five groups of simulation experiments. The nonlinear coefficient $\beta$ is set to 0.0001 , $0.0002,0.0003,0.0004$ and 0.0005 respectively. The waveform and stress nephogram of damaged material is similar to that of undamaged material because $\beta$ is relatively small.

The numerical simulation results are shown in Fig. 8 and Fig. 9. Different colours correspond to different $\beta$ values. When $\beta$ is determined (i.e. the damage degree of material is determined), we can see that the nonlinear detection coefficient of material increases with the increase of distance. The trend of increase is the same as when the material is undamaged. However, when the material is damaged, the nonlinear coefficient increases faster. This is a very obvious feature, the damage degree of two identical materials can be judged through it. We can get the reference value by measuring the undamaged material and then judge the damage degree of the material at a certain time by the reference value.

In Fig. 8 and Fig. 9, a virtual vertical line means a certain propagation distance. If the propagation distance is constant, the second detection coefficient $\beta^{\prime}$ is larger as the material damage is more serious.

In such a degree of mesh generation, the detection coefficient $\gamma^{\prime}$ obtained by measuring the third harmonic is highly sensitive to the damage of materials by comparing with $\beta^{\prime}$. However, $\beta^{\prime}$ is a better quantity to show material damage by comparison of Fig. 8 and Fig. 9. Because there is a more obvious linear relationship between $\beta^{\prime}$ and propagation distance. This linear relationship provides a basis for quantitative measurement of material damage in practical engineering.

From the spectrum in Fig. 5, we can see, the amplitude of the third harmonic is extremely small. If the simulation mesh is not dense enough, it is easy to produce large errors. In the actual engineering, the measurement is difficult and the error is large because the amplitude of the third harmonic is too small. So the second harmonic is still the main method for applying nonlinear ultrasound to practical engineering.

Through the above simulations and analysis, we can see that in the uniformly damaged material, or before the crack occurs, the regional damage of materials in early stage can be detected. 


\section{Conclusions}

Nonlinear respond of ultrasound is an important means to explore the damage degree of materials. As the mechanical properties of materials deteriorate, the constitutive relations of materials change. In this manuscript, the constitutive relation of simulation is changed by the method of writing a subroutine. It is found that early material degradation can be detected by ultrasound before the appearance of cracks. The generated harmonics are characterized by nonlinear detection coefficients $\beta^{\prime}$ and $\gamma^{\prime}$ : the nonlinear detection coefficient increases as the distance of propagation increases, reflecting the accumulation effect of harmonics. In addition, the nonlinear coefficient increases with the increase of material damage degree when the propagation distance is fixed. This laid a foundation for damage determination of materials in the early dislocation stage.

\section{References}

1. Hong Gun Kim, Hee Jae Shin, Sun Ho Go, Min Sang Lee, Alexandre Tugirumubano, Yoo Bin Kim, Kyoung Soo Kim, and Lee $\mathrm{Ku}$ Kwac, International Journal of Mechanical Engineering and Robotics Research, 6, 1 (2017)

2. C. R. Farrar and K. Worden, Philos Trans A Math Phys Eng Sci, 365, 1851 (2007)

3. N. G. Meyendorf, H. R“osner, V. Kramb, and S. Sathish, 40, 8, (2002)

4. Cantrell and J. H, Proceedings of the Royal Society A: Mathematical, Physical and Engineering Sciences, 460, 2043 (2004)
5. A. S. Birks, ASNT Handbook, 1991.

6. P. B. Nagy, Ultrasonics, 36, 5 (1998)

7. A. Raghavan, Dissertation Abstracts International, 975, 2 (2007)

8. I. Y. Solodov, N. Krohn, and G. Busse, Ultrasonics, 40, 1 (2002)

9. I. K. Zarembo and V. A. Krasil'Nikov, Soviet Physics Uspekhi, 102, 6 (1970)

10. K. Y. Jhang and K.-C. Kim, Ultrasonics, 37, 1 (1999)

11. Y. Zheng, R. G. Maev, and I. Y. Solodov, Canadian Journal of Physics, 77, 12 (1999)

12. D. Broda, W. Staszewski, A. Martowicz, T. Uhl, and V. Silberschmidt, Journal of Sound and Vibration, 333, 4 (2014)

13. K. Y. Jhang, IEEE-UFFC, 47, 3 (2000).

14. E. L.D. Landau, Oxford: Elsevier Ltd, 187 (1986)

15. K. E. A. V. D. Abeele and J. Carmeliet, Research in Nondestructive Evaluation, 12, 31-42 (2000).

16. B. O., Review of progress in quantitative nondestructive evaluation, 1990.

17. J. H. Cantrell and W. T. Yost, Philosophical Magazine A, 69, 2, (1994).

18. X. Wan, Q. Zhang, G. Xu, and P. Tse, Sensors, 14, 8528-8546 (2014)

19. G. M. Fierro, F. Ciampa, D. Ginzburg, E. Onder, and M. Meo, Journal of Sound and Vibration, 343, 121$130(2015)$

20. V. D. Abeele and K., The Journal of the Acoustical Society of America, 99, 3 (1995).

21. J. Jingpin, M. Xiangji, H. Cunfu, and W. Bin, NDT \& E International, 85, 63-71 (2016). 